\title{
Surface Plasmon-Driven Reversible Transformation of DNA-Bound Methylene Blue Detected In Situ by SERS
}

\author{
Muhammad R. Shattique $^{1} \cdot$ Maria Stepanova ${ }^{1,2}$ \\ Received: 30 April 2019 / Accepted: 30 September 2019 / Published online: 11 November 2019 \\ (C) The Author(s) 2019
}

\begin{abstract}
We have reported the in situ surface-enhanced Raman spectroscopy (SERS) monitoring of repetitive surface plasmon-mediated chemical transformation cycles in a conjugate nanobiological system. The nanobiological conjugate comprised a gold-coated plasmonic substrate biofunctionalized with thiolated single-stranded DNA carrying a reduction-oxidation indicator methylthioninium chloride, which is also known as methylene blue (MB), in buffer solution at a neutral $\mathrm{pH}$. Exposure to a 523-nm laser excitation produced pronounced SERS bands of oxidized MB. Continued exposure to the laser resulted in disappearance of the SERS bands, which can be interpreted as a reduction of MB. This occurred in the absence of electrochemical stimulation, chemical agents, or catalysts, suggesting a surface plasmon-mediated mechanism of the transformation. The oxidized form of MB was recovered by an addition of fresh buffer solution on the surface of the sample. Continued laser exposure with periodical addition of the buffer resulted in repetitive cycles of changes in the SERS pattern, which were monitored in situ. The chemical transformations of MB were preceded by a buildup of an intermediate SERS pattern, which was attributed to a transient form of MB created by selective surface plasmon-driven excitation.
\end{abstract}

Keywords Plasmon-driven photochemical reactions $\cdot$ SERS $\cdot$ Nanoplasmonics $\cdot$ Nanobiological conjugates $\cdot$ Methylene blue

\section{Introduction}

Engineering of multifunctional nanobioelectronic materials is a rapidly advancing interdisciplinary field of research and innovation. The integration of stimuli-responsive biological components with nanostructured surfaces of solid-state electronic devices is expected to revolutionize biomedical instrumentation, environmental diagnostics, and green energy harvesting technologies $[1,2]$. A potentially transformative pathway involves the usage of nanoplasmonics to both manipulate and monitor conjugate nanobiological architectures, while harvesting energy from visible light [3-6]. Surface plasmons are oscillations of electron density at a surface of a material. When a proper nanostructure (plasmonic substrate) is exposed to light, highly efficient absorption of incoming photons is possible, which is accompanied by a buildup of "hot-spots" - nano-sized regions

Maria Stepanova

ms1@ualberta.ca; mariastepanova@missouristate.edu

1 Department of Physics, Astronomy and Materials Science, Missouri State University, Springfield, MO 65897, USA

2 Department of Electrical and Computer Engineering, University of Alberta, Edmonton, AB T6G1H9, Canada where electromagnetic energy is accumulated due to the excitation of surface plasmon oscillations. This conversion of energy from light into highly localized hotspots imparts plasmonic substrates with unique functionalities. In particular, the recent discovery of heterogeneous photocatalysis at the interface of plasmonic substrates' surfaces and chemically responsive materials, which is mediated by energetic "hot" carriers that arise from the decay of surface plasmons [4-8] promises pathways for selective photocatalytic synthesis of materials that otherwise could not be achieved at ambient conditions [3-6]. Furthermore, plasmonic substrates are known to enhance inelastic Raman scattering of light by materials located nearby $[4$, $9,10]$. This dramatically increases the sensitivity of the Raman spectroscopy, which in turn allows capturing unique vibrational fingerprints of materials. The resulting characterization method, known as the surface-enhanced Raman spectroscopy (SERS), is highly regarded as a method of selective and ultrasensitive detection down to the submonolayer quantities of materials $[10,11]$. Importantly, most plasmonic substrates that show a strong SERS enhancement are also efficient as photocatalysts and vice versa.

This multifunctional nature of plasmonic substrates enables a unique platform that combines the capabilities of energy harvesting from visible light, photocatalytic functions, and 
ultrasensitive characterization of molecular events in the same design [4, 11-15]. The integration of plasmonic substrates with stimuli-responsive biological materials promises countless self-powered nanobiological systems with precise positional control of selectively stimulated molecular events. For this potential to be realized, specific conjugate architectures that allow for combined surface plasmon-driven photocatalytic stimulation and monitoring need to be designed and investigated. The development of such systems is progressing quickly. SERS in situ monitoring of surface plasmon-driven reduction-oxidation [15-17] or polymerization [18] processes has been reported recently.

In this work, we have developed a conjugate nanobiological system interfacing plasmonic gold nanostructures with thiolated single-stranded DNA carrying an important reduction-oxidation indicator, methylthioninium chloride $\mathrm{C}_{16} \mathrm{H}_{18} \mathrm{ClN}_{3} \mathrm{~S}$, which is also known as methylene blue (MB). The scheme of the conjugate system is outlined in Fig. 1. MB is well-known to adopt oxidized or reduced forms. In solution, at neutral or acidic $\mathrm{pH}, \mathrm{MB}$ adopts an oxidized form (denoted here as $\mathrm{MB}^{+}$), which produces a characteristic Raman spectrum $[19,20]$. Additionally, MB is known to bind to guanine bases [21, 22], which makes it a useful marker for nanobiological architectures involving DNA. We have investigated the evolution of SERS spectra of DNA-bound MB in our conjugate system under laser exposure in aquatic environment and captured a reversible reduction-oxidation process mediated by surface plasmons during the laser excitation.

\section{Materials and Methods}

Plasmonic nanostructures were created by magnetron deposition of thin (15 nm) gold coating on glass substrates using an Emitech K550X system from Quorum Technologies, with a deposition rate of $10 \mathrm{~nm} / \mathrm{min}$. Prior to the Au coating, the glass slides were cleaned thoroughly. The slides were first washed with acetone and DI water; then, they were placed in a Bransonic ultrasonic bath for $5 \mathrm{~min}$. After the sonication, each sample was nitrogen-dried. Finally, the slides were radiofrequency (RF) plasma-etched using a Jupiter III instrument (Nordson March). Etching was performed for $1 \mathrm{~min}$ with an $\mathrm{Ar} / \mathrm{H}_{2}$ mixture first, followed by a $30-\mathrm{s} \mathrm{H}_{2}$ etching.

We employed a Bruker Dimension Icon atomic force microscopy (AFM) instrument to characterize our gold-coated glass substrates. Figure 2 presents the surface morphology of Au coating with a $15 \mathrm{~nm}$ average thickness on a glass support. Nanosized self-assembled islands of Au are clearly seen in the figure. Tiny gaps around such islands are expected to provide plasmonic hotspots when the substrate is exposed to light [9-11, 23].

The Au-coated substrates were biofunctionalized as schematically shown in Fig. 1. For this purpose, lyophilized thiolated DNA material, 5'-GGTTTGGAGGGGC [SpcC3]Thiol, and an $1.5 \% \mathrm{MB}$ solution were purchased from Sigma-Aldrich. The DNA sequence is one of natural guanine-rich genome fragments (see, e.g., Ref. [24]). A $1 \mu \mathrm{M}$ DNA solution was prepared with Tris-EDTA buffer at a $\mathrm{pH}$ of 7 . The solution was stored at $5{ }^{\circ} \mathrm{C}$. To biofunctionalize the samples, a $100 \mu \mathrm{L}$ drop of the DNA solution was deposited onto the gold-coated substrates. Next, a $100 \mu \mathrm{L}$ drop of a 4.7 $\mathrm{mM}$ MB solution was added onto the same spot, and the samples were incubated in humid environment for $24 \mathrm{~h}$ at $5^{\circ} \mathrm{C}$ as illustrated elsewhere [25]. It was expected that during the incubation, DNA strands would bind to the surface of gold coating through thiol groups [23, 26, 27], whereas MB would bind to guanine bases of the DNA [21, 22].

After the incubation, the samples were thoroughly rinsed in the Tris-EDTA buffer with a $\mathrm{pH}$ of 7, and a drop of fresh buffer was deposited onto their surface. The volume of the
Fig. 1 Scheme of the conjugate nanobiological system interfacing MB-loaded, thiolated DNA with Au-coated plasmonic substrate in an aquatic environment

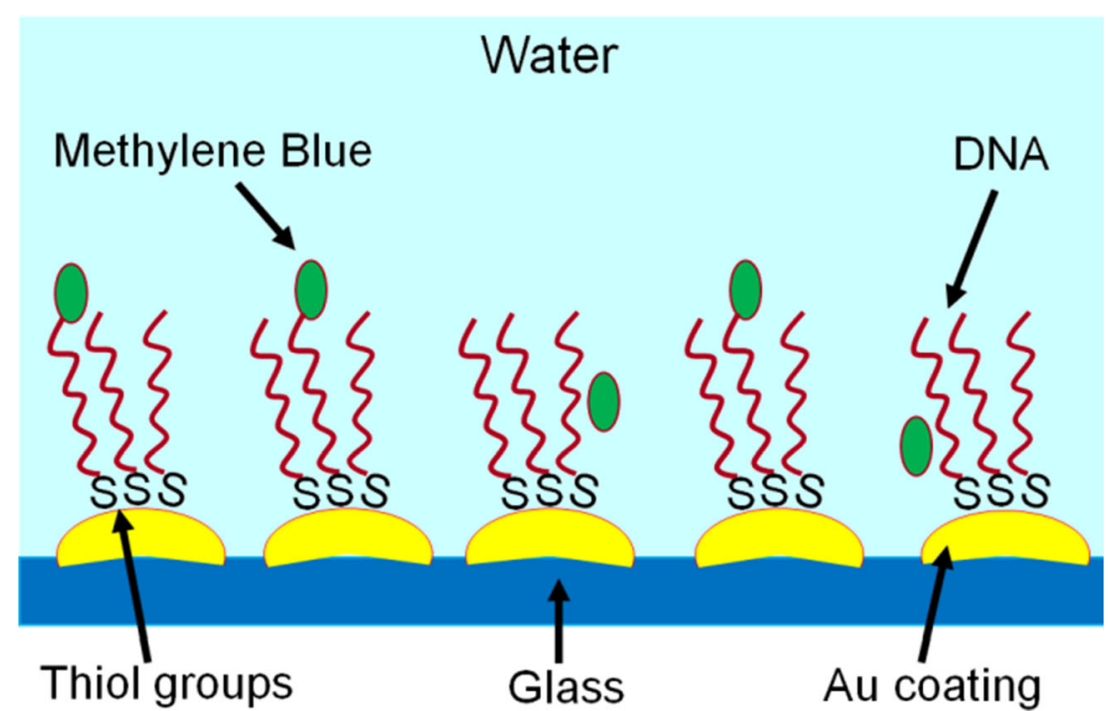




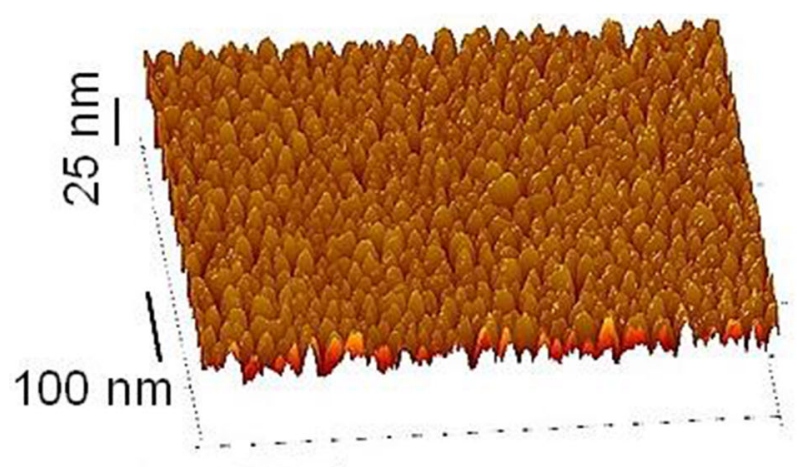

(a)

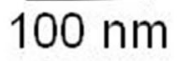

(b)

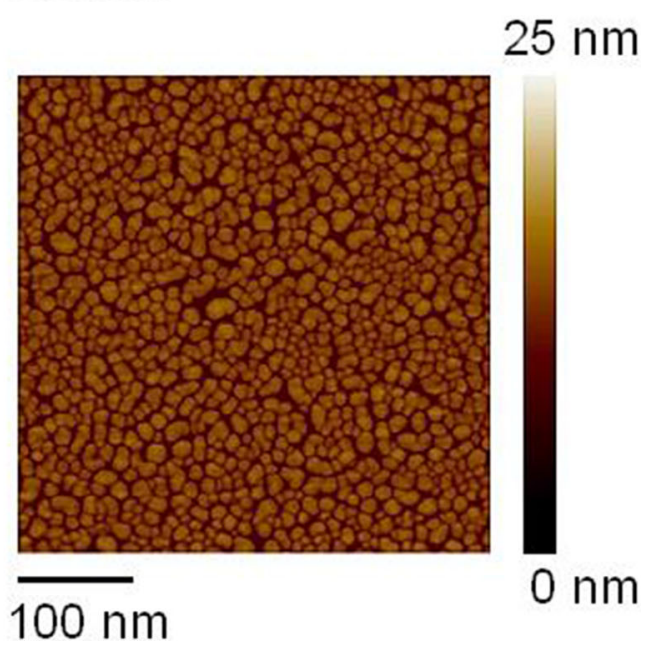

Fig. 2 AFM surface morphology of the Au-coated glass substrates: a 3D topographical image; b plan-view image.

drop was adjusted to keep the biofunctionalized surfaces in liquid environment during subsequent laser exposures (Fig. 3). For the laser exposures and acquisition of the Raman spectra, a Horiba LabRAM HR800 Evolution Raman microspectroscope was employed. We used a $523 \mathrm{~nm}$ excitation wavelength with a laser power of $50 \mathrm{~mW}$, and a $10 \mathrm{x}$ magnification. Care was taken that a drop of liquid remains on the surface of the samples during all the exposures.

For benchmarking purposes, control samples comprising a $100 \mu \mathrm{L}$ drop of the $4.7 \mathrm{mM} \mathrm{MB}$ solution deposited directly onto gold-coated glass substrates in the absence of DNA were also characterized. Two kinds of such samples were prepared. Control samples \#1 were characterized immediately after the deposition of MB solution onto their surface, without incubation or rinsing. Control samples \#2 were subjected to similar incubation and rinsing procedures as those containing both $\mathrm{MB}$ and DNA. A different set of control samples, \#3, comprised $100 \mu \mathrm{L}$ drops of MB solution onto bare uncoated glass slides without gold coating. In one more set of control samples, \#4, drops of both MB and DNA solutions were deposited onto the surface of bare glass slides without gold coating. Finally, in control samples \#5, DNA solution without MB was incubated on gold-coated glass slides.

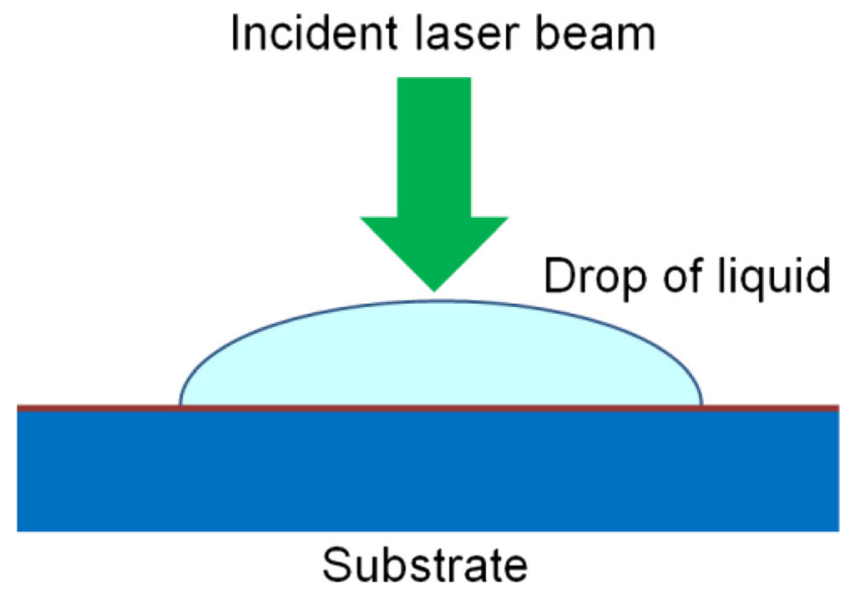

Fig. 3 Scheme of the samples during laser exposures

\section{Results and Discussion}

Figures. $4 \mathrm{a}$ and $\mathrm{b}$ present the typical Raman spectra of control samples \#1 and \#2, respectively. Fig. 4a, which represents a spectrum of the MB solution immediately after deposition onto gold-coated substrate in the absence of DNA, exhibits pronounced bands attributable to $\mathrm{MB}^{+}$in the oxidized form. Based on published literature [19, 20], we expect the strongest band at approximately $1625 \mathrm{~cm}^{-1}$ to originate from stretching of C-C bonds of MB's aromatic rings, those between 1398 $\mathrm{cm}^{-1}$ and $1470 \mathrm{~cm}^{-1}$ to represent vibrations of $\mathrm{N}-\mathrm{C}$ bonds and $\mathrm{CH}_{3}$ groups, and those at $501 \mathrm{~cm}^{-1}$ and $446 \mathrm{~cm}^{-1}$ to result from $\mathrm{C}-\mathrm{N}-\mathrm{C}$ skeletal bending.

Figure $4 \mathrm{~b}$ depicts a spectrum of a similar MB solution after a 24-h incubation in the Tris-EDTA buffer at a $\mathrm{pH} 7$ on a gold-coated glass slide in the absence of DNA and subsequent rinsing. The spectrum does not exhibit any bands attributable to $\mathrm{MB}$, indicating that the MB molecules do not attach to the gold-coated substrate's surface strongly enough to remain in a detectable quantity after rinsing. Spectra from the DNA incubated on gold-coated glass slides in the absence of $\mathrm{MB}$ were also acquired. No pronounced signal that could interfere with the MB fingerprint was detected.

Figure 5 shows an example spectrum of a sample incubated with both DNA and MB after subsequent rinsing. In striking contrast with the DNA-free samples, pronounced SERS bands typical of oxidized $\mathrm{MB}^{+}$are visible in this figure. These include a strong peak at approximately $1623 \mathrm{~cm}^{-1}$. We have concluded that during the incubation, thiolated and MBloaded DNA binds to the surface of gold, playing a role of surface-immobilized linker for MB. Remarkably, after rinsing of the samples, surface-immobilized DNA is supposed to be present only in a monolayer quantity or less. Therefore, pronounced SERS fingerprints such as shown in Fig. 5 correspond to an extremely small quantity of DNA-bound $\mathrm{MB}^{+}$ molecules. This suggests a significant signal enhancement, 


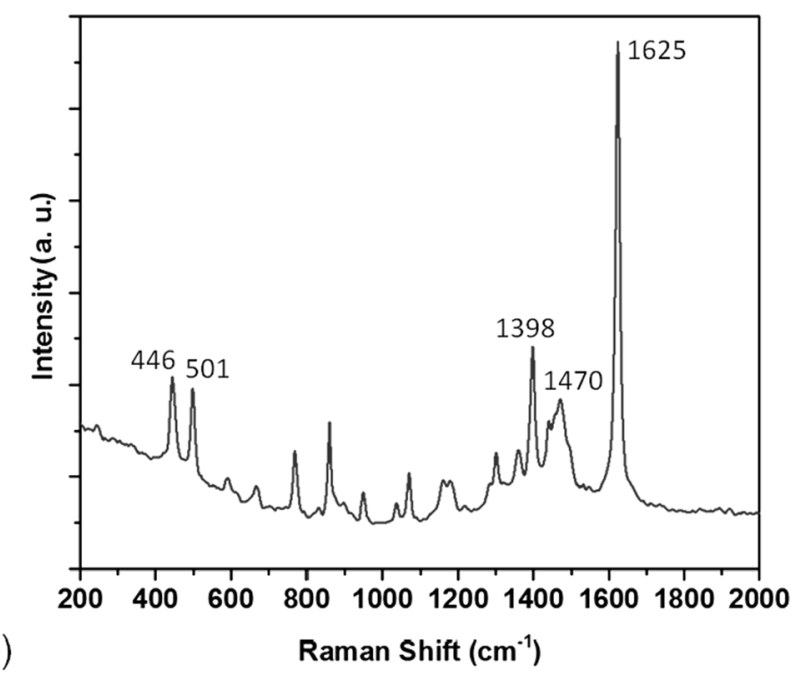

(a)

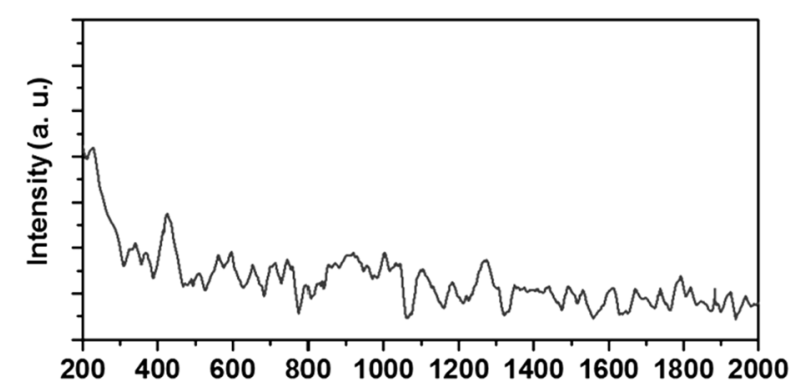

(b)

Raman Shift $\left(\mathrm{cm}^{-1}\right)$

Fig. 4 Raman spectra of control samples containing methylene blue on gold-coated substrates in the absence of DNA [25]: a spectrum of $\mathrm{MB}^{+}$ solution on the substrate without incubation and rinsing; $\mathbf{b}$ spectrum after deposition of the MB solution on the substrate, 24-h incubation, and rinsing

indicating that self-assembled Au nano-islands seen in Fig. 2 have been efficient enough for a buildup of plasmonic hotspots under the 523-nm laser excitation.

Next, we have collected the SERS spectra of the samples carrying MB-loaded DNAs after exposures to the laser excitation of various durations. Figure 6 compares the SERS spectrum obtained at the beginning of the exposure of the sample with those acquired after longer exposures. Initially, at the beginning of the experiment, a fingerprint of oxidized $\mathrm{MB}^{+}$ was clearly visible. However, after $100 \mathrm{~min}$ of exposure to the laser, the SERS fingerprint completely disappeared. At that point, we added a $20 \mu \mathrm{L}$ drop of fresh Tris-EDTA buffer $(\mathrm{pH} 7)$ onto the same sample and continued the laser exposure. This resulted in a recovery of SERS fingerprint of $\mathrm{MB}^{+}$. After $140 \mathrm{~min}$ of exposure, a clear band was visible at $1630 \mathrm{~cm}^{-1}$. To further investigate this phenomenon, we carried out the experiment for a longer time. As Fig. 6 illustrates, continuing the laser exposure resulted in the disappearance of the $\mathrm{MB}^{+}$ bands after $180 \mathrm{~min}$, whereas repeated addition of the buffer led to their recovery. After $240 \mathrm{~min}$ of the exposure, a band from the vibrations of $\mathrm{MB}^{+}$aromatic rings centered at 1624

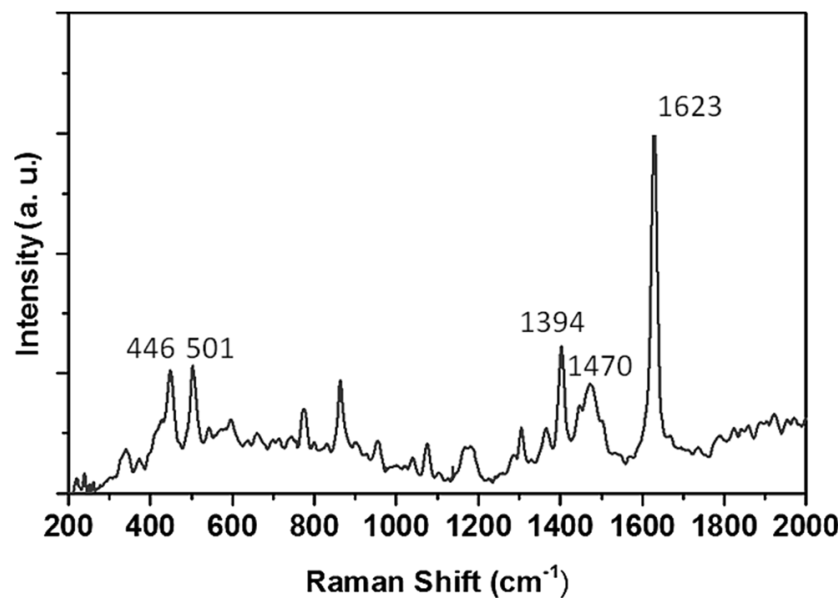

Fig. 5 SERS spectrum of a sample carrying MB-loaded DNA immobilized on gold-coated glass substrate after 24-h incubation and subsequent rinsing [25]

$\mathrm{cm}^{-1}$ was observed again. The sample's surface remained humid at all times during these experiments.

Degradation and/or disappearance of Raman bands characteristic of oxidized $\mathrm{MB}^{+}$was found previously in electrochemical experiments, when negative electrode potential was

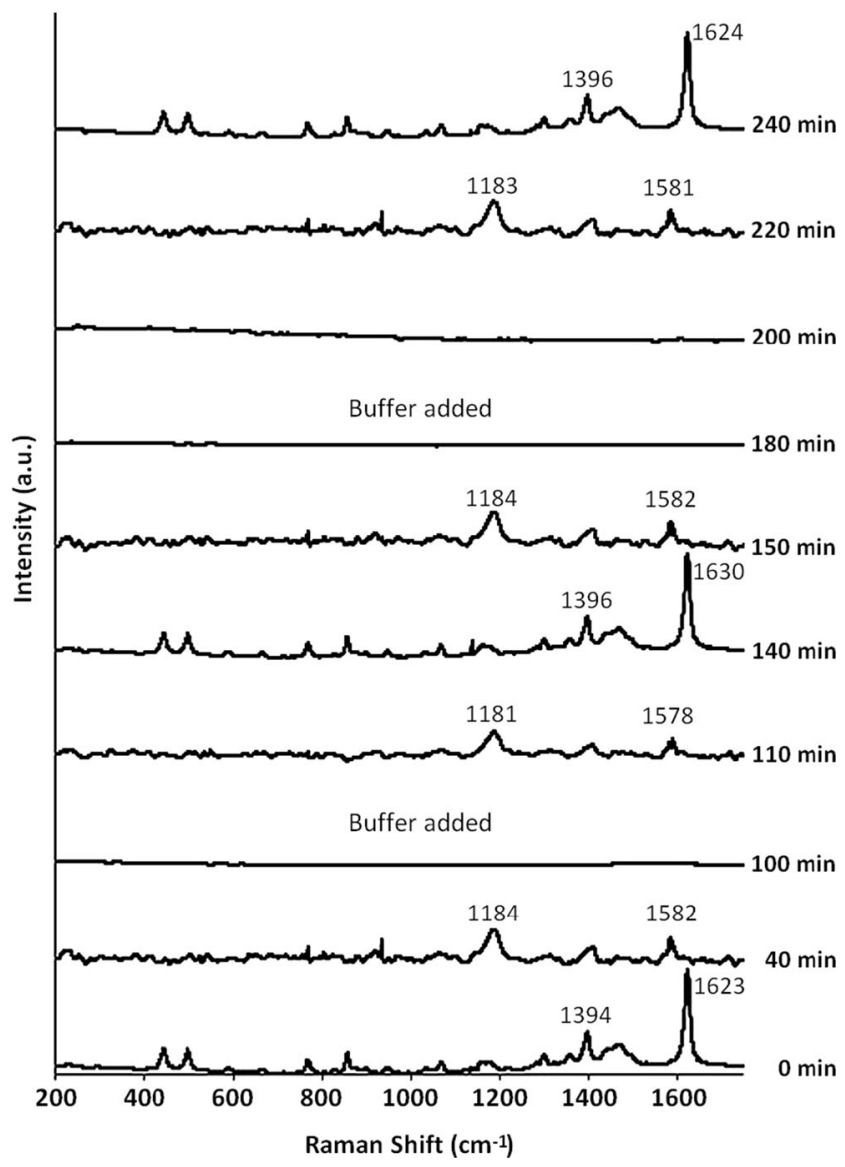

Fig. 6 SERS spectra of a sample carrying MB-loaded immobilized DNA after various durations of exposure to a $523-\mathrm{nm}$ laser. Fresh Tris-EDTA buffer was added after $100 \mathrm{~min}$ and $180 \mathrm{~min}$ of the exposure, as indicated. A vertical offset was applied to the spectra for clearer presentation 
applied to an MB solution [20, 28] or immobilized MB-loaded DNA [29]. Upon the reversal of the applied potential, the recovery of the MB's Raman fingerprint was observed [29]. Thus, the absence of SERS bands at $100 \mathrm{~min}$ and $180 \mathrm{~min}$ of laser exposure, as illustrated in Fig. 6, is attributable to a reduction of DNA-bound MB, whereas recovery of the spectrum at $140 \mathrm{~min}$ and $240 \mathrm{~min}$ can be interpreted as its reversible oxidation. However, unlike the earlier studies, the cyclic changes of the SERS spectra, as seen in Fig. 6, occurred in the absence of electrochemical stimulation.

Remarkably, both the disappearance of $\mathrm{MB}^{+}$SERS fingerprint and its recovery, as seen in Fig. 6, are preceded by a buildup of an alternative SERS pattern. As Fig. 6 illustrates, the spectra acquired after $40 \mathrm{~min}, 110 \mathrm{~min}, 150 \mathrm{~min}$, and $220 \mathrm{~min}$ of laser exposure are dissimilar to that at the beginning of the experiment as well as those seen at 140 and $240 \mathrm{~min}$ of the exposure. Additionally, the examples of the transition spectra are presented in Fig. 7. Instead of a strong $1623-1630 \mathrm{~cm}^{-1}$ band representing vibrations of $\mathrm{MB}^{+}$'s aromatic rings, a smaller but pronounced peak is observed at $1578-1582 \mathrm{~cm}^{-1}$. According to the literature [19], this band is still attributable to vibrations of aromatic rings in $\mathrm{MB}$, although the shift in position indicates a decrease in frequency of these vibrations. Another pronounced band is found at $1181-1184 \mathrm{~cm}^{-1}$. A similarly positioned but relatively weak band is discernible in the $\mathrm{MB}^{+}$fingerprints (see, e.g., Figs. 4a and 5), which can be attributed to $\mathrm{N}-\mathrm{C}$ bonds stretching in MB [19]. We have hypothesized that the alternative SERS spectrum originates from DNA-bound MB in an intermediate transition state, which we have denoted as MB*.

To elucidate the origin of the observed reductionoxidation transformations of $\mathrm{MB}$, we have carried out similar experiments with control samples \#3, which comprised a drop of $\mathrm{MB}$ solution deposited onto bare glass slides without gold coating and DNA functionalization. The samples were subjected to laser exposures as described above. As Fig. 8 illustrates, in this case, a characteristic Raman band of oxidized $\mathrm{MB}^{+}$, centered at approximately $1629 \mathrm{~cm}^{-1}$, has remained after $100 \mathrm{~min}$ of laser exposure. Furthermore, subsequent addition of $20 \mu \mathrm{L}$ of Tris-EDTA buffer did not change the Raman fingerprint. Additionally, control samples \#4, which carried drops of both MB and DNA solutions on an uncoated glass slide, were characterized. The corresponding Raman spectra were similar to those shown in Fig. 8 and remained stable during laser exposures.

The absence of reduction-oxidation transformations in MB samples on bare uncoated glass surface indicates that the proximity to the nanostructured gold coating (Fig. 2) is critical for these transformations. We have inferred that surface-plasmonic waves generated by laser excitation in the gold coating are required for the chemical transformations of $\mathrm{MB}$ to occur. The fact that the observed transformations occur in a solution of initially neutral $\mathrm{pH}$, in the absence of electrochemical stimulation, and without catalysts other than the gold coating itself speaks in favor of surface plasmon-driven mechanism of the transformations.

Our SERS results suggest that both photochemical reduction and subsequent oxidation of MB occur through an intermediate transition state MB*, which is different from both the two generic forms of MB. It is well-known that electrochemical $[20,28]$ or photochemical $[30,31]$ reduction of $\mathrm{MB}$ are multistep processes and may involve several intermediate states. As a consequence, the corresponding infrared [30] or Raman [28] spectra typically express a rich pattern consisting of many overlapping bands from various reaction products. Although the Raman bands close to $1578-1582 \mathrm{~cm}^{-1}$ and $1181-1184$ $\mathrm{cm}^{-1}$ regions, as we observe for $\mathrm{MB}^{*}$, have been reported after electrochemical reduction of MB solution [20, 28 ], they typically were a part of complex multiband patterns. The relatively simple Raman spectra as we find for $\mathrm{MB}^{*}$, to the best of our knowledge, have not been observed. The simplicity of the Raman fingerprints observed after several repetitive reduction-oxidation cycles suggests that photothermal effects are unlikely to be the leading mechanism of the changes in the spectra. In case of thermal excitation, a wide range of the intermediate products would be created, with a low likelihood of such simple Raman fingerprints as observed here. Even more intriguing is the similarity of $\mathrm{MB}^{*}$ spectra for the reduction and oxidation processes.

These findings might be rationalized under the assumption of plasmonic-driven photocatalytic mechanism behind the observed transformations of MB. In this mechanism, the surface-plasmonic waves induced by light absorption in metal nanostructures decay through the creation of transient energetic charge carriers of both signs at the interface of metal and the adsorbate $[4-6,8]$. These transient "hot electrons" or "hot holes" may be injected directly into specific electronic states of the adsorbate through the mediation of strong electric fields in plasmonic hotspots $[5,6,8,17]$. This, in turn, opens a possibility of inducing highly selective chemical transformations in materials adsorbed near the hotspots, which otherwise would be impossible at ambient conditions $[5,8,15]$. We have anticipated that the transient MB* form might be selectively excited by direct injection of plasmonic hot carriers with the mediation of enhanced electromagnetic field in the hotspots and subsequently transformed into either reduced or oxidized $\mathrm{MB}$, depending on the availability of sources/sinks of proper charge carriers in the samples. As an addition of fresh buffer is required to trigger the reversible oxidation of $\mathrm{MB}$, the carriers can be expected to originate from the buffer. A possibility of direct plasmon-driven excitation of MB molecules attached to Ag nanoparticles has been demonstrated recently [5]. Our findings suggest that a highly selective, direct excitation might 
Fig. 7 The SERS transition spectra at $40 \mathrm{~min}$ of laser exposure (a) and $110 \mathrm{~min}$ of exposure (b)
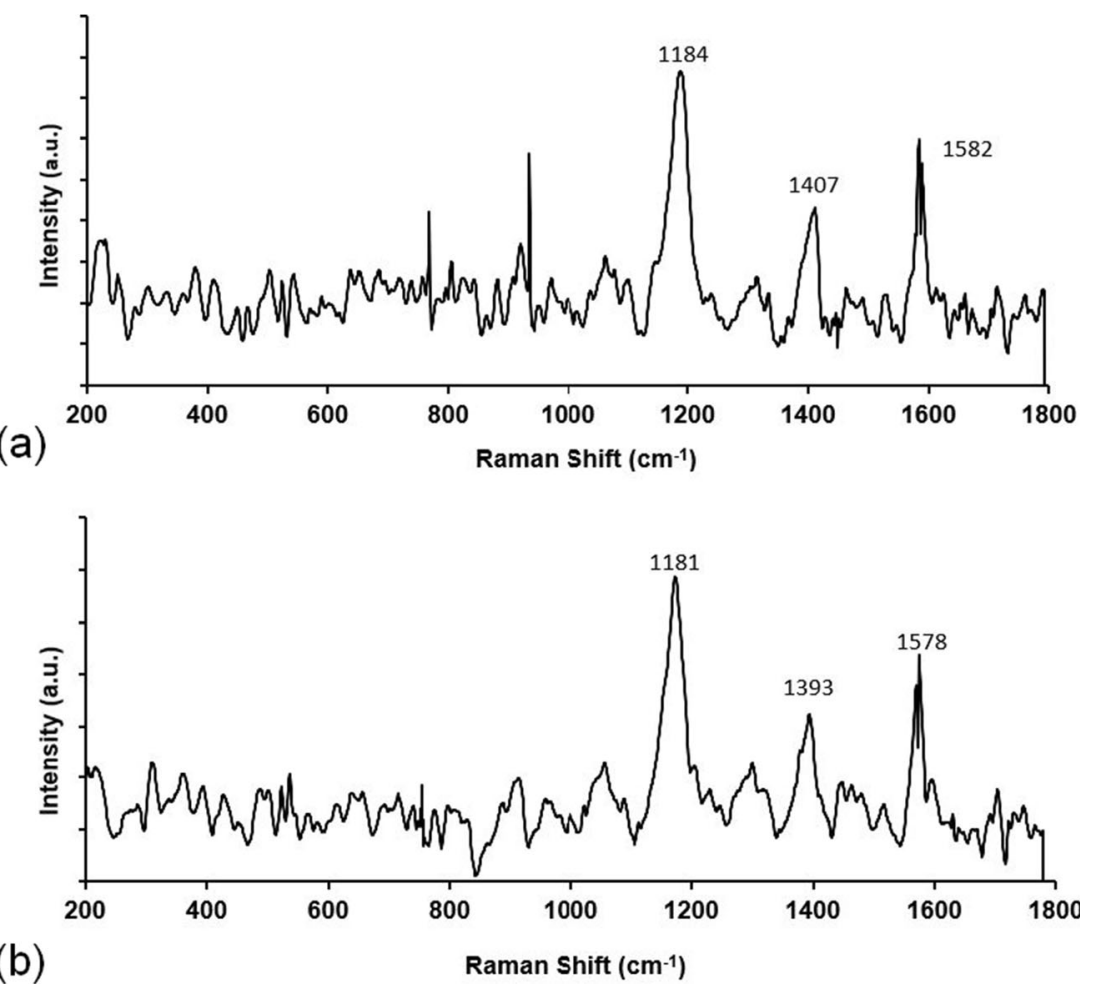

be possible in MB attached to DNA and immobilized on a surface of plasmonic $\mathrm{Au}$ nanostructures in an aquatic environment.

\section{Summary}

We used a simple method, the self-assembly of $\mathrm{Au}$ nano-islands by magnetronic deposition onto glass supports, to fabricate plasmonic substrates. We biofunctionalized these substrates with thiolated MB-loaded DNA and investigated the response of the samples to a 523-nm laser exposures while keeping the samples in an aquatic environment during the entire experiment. Shortly after being subjected to the laser exposure, the samples exhibited pronounced SERS bands of oxidized MB.

Continued exposure to the laser with periodical addition of fresh buffer solution at a neutral $\mathrm{pH}$ resulted in repetitive cycles of reversible reduction-oxidation of DNA-bound MB, which were monitored by SERS in situ. The transformations occurred in the absence of electrochemical stimulation, chemical agents, or catalysts. However, plasmonic gold coating was
Fig. 8 The Raman spectra of a drop of MB solution ( $\mathrm{pH} 7$ ) on bare uncoated glass slide at the beginning of laser exposure (bottom); after 100 min of the exposure (middle); and after $140 \mathrm{~min}$ of the exposure following the addition of Tris-EDTA buffer (top). A vertical offset was applied to the spectra for clearer presentation

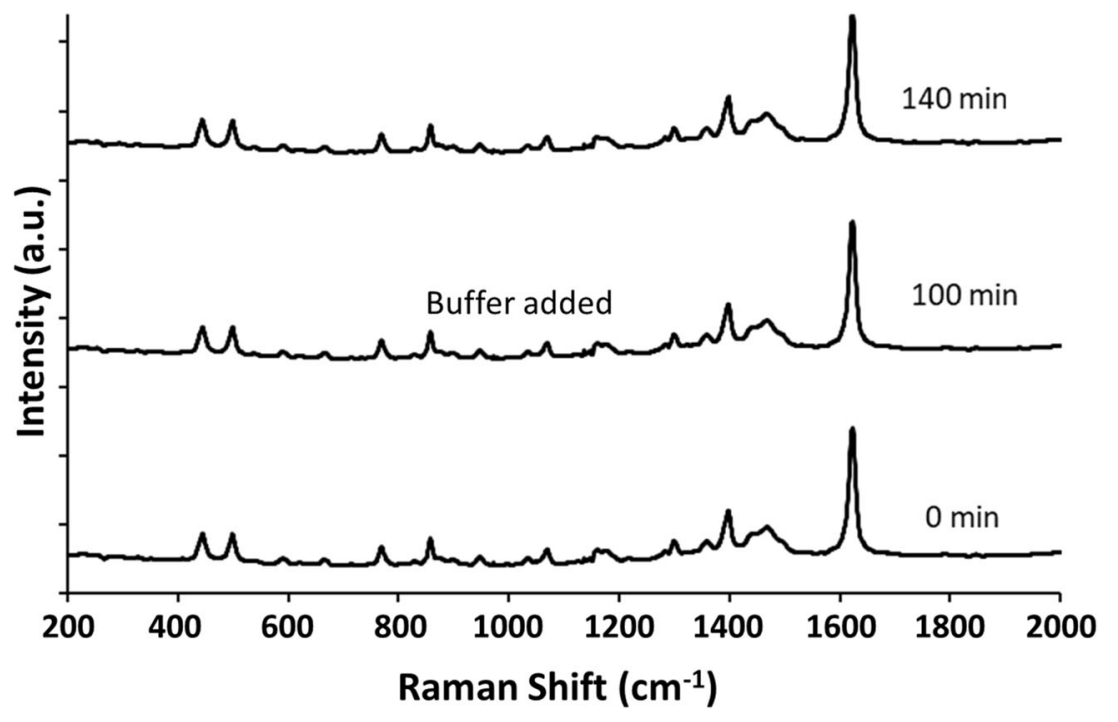


required for the transformations to occur, suggesting a surface plasmon-mediated mechanism. Both the reduction and oxidation of MB were preceded by a buildup of an intermediate SERS pattern, which was attributed to a transient form of MB created by surface plasmon-driven direct excitation.

Due to multifunctional nature of plasmonic substrates, the simple design described here allowed simultaneous harvesting of energy from laser exposures, inducing specific chemical transformations in DNA-bound MB, and detecting these transformations in situ from submonolayer quantities of MB-loaded DNA material. Clearly, further research is required to unravel details of these transformations. We expect that the multifunctional nanobiological system described here will be useful for future research on surface plasmon-driven photocatalysis, elucidating the reaction mechanisms involved, optimizing plasmonic substrates, and refining their applications.

Acknowledgements The authors would like to thank Dr. R. Mayanovic for providing access to the LabRAM instrument and his helpful advises. We are also grateful to Rishi Patel and staff of the Roy Blunt Jordan Valley Innovation Center (JVIC) for their assistance in the preparation of gold-coated substrates and AFM characterization. Support of the work by the Department of Physics, Astronomy, and Materials Science of the Missouri State University is gratefully acknowledged.

\section{Compliance with Ethical Standards}

Conflict of Interest The authors are not aware of a conflict of interest to disclosure.

Abbreviations AFM, atomic force microscopy; MB, methylene blue; $\mathrm{RF}$, radiofrequency; SERS, surface-enhanced Raman spectroscopy

Open Access This article is distributed under the terms of the Creative Commons Attribution 4.0 International License (http:// creativecommons.org/licenses/by/4.0/), which permits unrestricted use, distribution, and reproduction in any medium, provided you give appropriate credit to the original author(s) and the source, provide a link to the Creative Commons license, and indicate if changes were made.

\section{References}

1. Ross AM, Lahann J (2015) Current trends and challenges in biointerfaces science and engineering. Annu Rev Chem Biomol 6:161-186

2. McKeating KS, Aubé A, Masson JF (2016) Biosensors and nanobiosensors for therapeutic drug and response monitoring. Analyst 141:429-449

3. Naldoni A, Shalaev VM, Brongersma ML (2017) Applying plasmonics to a sustainable future. Science 356:908-909

4. Zhan C, Chen XJ, Yi J, Li JF, Wu DY, Tian ZQ (2018) From plasmon-enhanced molecular spectroscopy to plasmon-mediated chemical reactions. Nat Rev Chem 2:216-230
5. Aslam U, Rao VG, Chavez S, Linic S (2018) Catalytic conversion of solar to chemical energy on plasmonic metal nanostructures. Nat Catal 1:656-665

6. Linic S, Aslam U, Boerigter C, Morabito M (2015) Photochemical transformations on plasmonic metal nanoparticles. Nat Mater 14: $567-576$

7. Wang C, Astruc D (2014) Nanogold plasmonic photocatalysis for organic synthesis and clean energy conversion. Chem Soc Rev 43: 7188-7216

8. Zhang Y, He S, Guo W, Hu Y, Huang J, Mulcahy JR, Wei WD (2017) Surface-plasmon-driven hot electron photochemistry. Chem Rev 11:2927-2954

9. Anker JN, Hall WP, Lyandres O, Shah NC, Zhao J, Van Duyne RP (2008) Biosensing with plasmonic nanosensors. Nat Mater 7:442453

10. Ding SY, Yi J, Li JF, Ren B, Wu DY, Panneerselvam R, Tian ZQ (2016) Nanostructure-based plasmon-enhanced Raman spectroscopy for surface analysis of materials. Nat Rev Mater 1:16021

11. Cardinal MF, Ende EV, Hacker RA, McAnally MO, Stair PC, Schatz GC, Van Duyne RP (2017) Expanding applications of SERS through versatile nanomaterials engineering. Chem Soc Rev 46:3886-3903

12. Zrimsek AB, Chiang N, Mattei M, Zaleski S, McAnally MO, Chapman CT, Henry AI, Schatz GC, Van Duyne RP (2017) Single-molecule chemistry with surface- and tip-enhanced Raman spectroscopy. Chem Rev 117:583-7613

13. Polman A, Knight M, Garnett EC, Ehrler B, Sinke WC (2016) Photovoltaic materials: present efficiencies and future challenges. Science 352: $\operatorname{aad} 4424$

14. Liu R, He Z, Sun J, Liu J, Jiang G (2016) Tracking the fate of surface plasmon resonance-generated hot electrons by in situ SERS surveying of catalyzed reaction. Small 12:6378-6387

15. Ren X, Cao E, Lin W, Song Y, Liang W, Wang J (2017) Recent advances in surface plasmon-driven catalytic reactions. RSC Adv 7: 31189-31203

16. Cui L, Wang P, Fang Y, Li Y, Sun M (2015) A plasmon-driven selective surface catalytic reaction revealed by surface-enhanced Raman scattering in an electrochemical environment. Sci Rep 5: 11920

17. Cortés E, Xie W, Cambiasso J, Jermyn AS, Sundararaman R, Narang P, Schlücker S, Maier SA (2017) Plasmonic hot electron transport drives nano-localized chemistry. Nat Commun 8:14880

18. Kang L, Xu P, Zhang B, Tsai H, Han X, Wang HL (2013) Laser wavelength- and power-dependent plasmon-driven chemical reactions monitored using single particle surface enhanced Raman spectroscopy. Chem Commun 49:33893391

19. Xiao G-N, Man S-Q (2007) Surface-enhanced Raman scattering of methylene blue adsorbed on cap-shaped silver nanoparticles. Chem Phys Lett 447:305-309

20. Nicolai SHA, Rubim JC (2003) Surface-enhanced resonance Raman (SERR) spectra of methylene blue adsorbed on a silver electrode. Langmuir 19:4291-4294

21. Rohs R, Sklenar H, Lavery R, Röder B (2000) Methylene blue binding to DNA with alternating GC base sequence: a modeling study. J Am Chem Soc 122:2860-2866

22. Farzami E, Clima L, Gothelf K, Ferapontova EE (2010) DNA interactions with a methylene blue redox indicator depend on the DNA length and are sequence specific. Analyst 135:1443-1448

23. Peters RF, Gutierrez-Rivera L, Dew SK, Stepanova M (2015) Surface enhanced Raman spectroscopy detection of biomolecules using EBL fabricated nanostructured substrates. J Vis Exp 97: 52712

24. Swalla BJ Zeng L (2006) Boltenia villosa cytochrome oxidase B (COB) gene, partial cds; mitochondrial. GenBank, accession number DQ345907 
25. Shattique MR, Stepanova M (2018) SERS detection of reduction-oxidation processes in DNA-bound methylthioninium chloride on gold coated substrates. TechConnect Briefs, 3, 221-224, https://briefs.techconnect. org/wp-content/volumes/TCB2018v3/pdf/446.pdf. Accessed 4 June 2018

26. Love JC, Estroff LA, Kriebel JK, Nuzo RG, Whitesides GM (2005) Self-assembled monolayers of thiolates on metals as a form of nanotechnology. Chem Rev 105:1103-1169

27. Pensa E, Cortés E, Corthey G, Carro P, Vericat C, Fonticelli MH, Benitez G, Rubert AA, Salvarezza RC (2012) The chemistry of the sulfur-gold interface: in search of a unified model. Acc Chem Res 45:1183-1192

28. de Araujo Nicolai SE, Rodrigues PRP, Agostinho SML, Rubim JC (2002) Electrochemical and spectroelectrochemical (SERS) studies of the reduction of methylene blue on a silver electrode. J Electroanal Chem 527:103-111
29. Papadapoulou E, Gale N, Thompson JF, Fleming TA, Brown T, Bartlett NP (2016) Specifically horizontally tethered DNA probes on $\mathrm{Au}$ surfaces allow labelled and label-free DNA detection using SERS and electrochemically driven melting. Chem Sci 7:386-393

30. Yu Z, Chuang SSC (2007) Probing methylene blue photocatalytic degradation by adsorbed ethanol with in situ IR. J Phys Chem C 111:13813-13820

31. Houas A, Lachheb H, Ksibi M, Elaloui E, Guillard C, Herrmann JM (2001) Photocatalytic degradation pathway of methylene blue in water. Appl Catal B 31:145-157

Publisher's Note Springer Nature remains neutral with regard to jurisdictional claims in published maps and institutional affiliations. 